\title{
Supplementary Material: Accounting for Smoking in Forecasting Mortality and Life Expectancy
}

\author{
Yicheng Li and Adrian E. Raftery
}

November 17, 2019

\section{MCMC Convergence Diagnostics}

We check the convergence of BHM for ASSAF based on trace plots and diagnostics (Raftery and Lewis, 1992) for the global parameters in Level 3. We check one chain with 2,000 burn in iterations and 100,000 samples, with a thinning period of 20 iterations. Table1 1 shows the summary statistics of the diagnostics. Figure 1 shows the trace plots of all 3,000 samples of the global parameters.

We do the same for $e_{0}^{N S}$. We check one of the 30 samples with 1,000 burn in iterations, 100,000 samples and a thinning period of 50 iterations. Table 2 shows the summarizing statistics of the diagnostics. Figure 2 shows the trace plots of all 1,000 samples of the global parameters.

Table 1: Diagnostic statistics for global parameters in BHM for ASSAF. Burn1, Size1, and DF1 are the length of burn-in, required sample size, and dependence factor of the Raftery-Lewis diagnostics with parameters $q=0.025, r=0.0125$, and $s=0.95$. Burn2, Size2, and DF2 are the same, but for $q=0.975$.

\begin{tabular}{|c|c|c|c|c|c|c|}
\hline Parameters & Burn1 & Size1 & DF1 & Burn2 & Size2 & DF2 \\
\hline$\mu_{40}^{2[\beta]}$ & - & - & - & - & - & - \\
$\mu_{45}^{2[\beta]}$ & 2 & 606 & 1.01 & 2 & 631 & 1.05 \\
$\mu_{50}^{2[\beta]}$ & 2 & 641 & 1.07 & 2 & 581 & 0.97 \\
$\mu_{55}^{2[\beta]}$ & 2 & 577 & 0.96 & 2 & 631 & 1.05 \\
$\mu_{60}^{2[\beta]}$ & 2 & 616 & 1.03 & 2 & 591 & 0.98 \\
$\mu_{65}^{2[\beta]}$ & 2 & 601 & 1.00 & 2 & 621 & 1.03 \\
$\mu_{70}^{2[\beta]}$ & 2 & 616 & 1.03 & 2 & 621 & 1.03 \\
$\mu_{75}^{2[\beta]}$ & 2 & 587 & 0.98 & 2 & 611 & 1.02 \\
$\mu_{80}^{2[\beta]}$ & 2 & 626 & 1.04 & 3 & 664 & 1.11 \\
$\sigma_{40}^{2[\beta]}$ & - & - & - & - & - & - \\
$\sigma_{45}^{2[\beta]}$ & 3 & 669 & 1.12 & 3 & 653 & 1.09 \\
$\sigma_{50}^{2[\beta]}$ & 2 & 601 & 1.00 & 2 & 601 & 1.00 \\
$\sigma_{55}^{2[\beta]}$ & 2 & 591 & 0.98 & 2 & 621 & 1.03 \\
$\sigma_{60}^{2[\beta]}$ & 2 & 606 & 1.01 & 2 & 572 & 0.95 \\
$\sigma_{65}^{2[\beta]}$ & 2 & 611 & 1.02 & 2 & 611 & 1.02 \\
\hline
\end{tabular}


Table 1: Diagnostic statistics for global parameters in BHM for ASSAF. Burn1, Size1, and DF1 are the length of burn-in, required sample size, and dependence factor of the Raftery-Lewis diagnostics with parameters $q=0.025, r=0.0125$, and $s=0.95$. Burn2, Size2, and DF2 are the same, but for $q=0.975$.

\begin{tabular}{|c|c|c|c|c|c|c|}
\hline Parameters & Burn1 & Size1 & DF1 & Burn2 & Size2 & DF2 \\
\hline$\sigma_{70}^{2[\beta]}$ & 1 & 595 & 0.99 & 2 & 591 & 0.98 \\
$\sigma_{75}^{2[\beta]}$ & 3 & 648 & 1.08 & 2 & 641 & 1.07 \\
$\sigma_{80}^{2[\beta]}$ & 2 & 621 & 1.03 & 3 & 676 & 1.13 \\
$\sigma$ & 2 & 591 & 0.98 & 3 & 658 & 1.10 \\
$\sigma^{2[\tau]}$ & 2 & 591 & 0.98 & 3 & 648 & 1.08 \\
$\mu_{\triangle_{1}}$ & 6 & 1308 & 2.18 & 9 & 2040 & 3.40 \\
$\mu_{\triangle_{2}}$ & 8 & 1610 & 2.68 & 2 & 641 & 1.07 \\
$\sigma_{\triangle_{2}}^{2}$ & 12 & 2160 & 3.60 & 8 & 1586 & 2.64 \\
$\mu_{\triangle_{3}}$ & 4 & 790 & 1.32 & 3 & 686 & 1.14 \\
$\mu_{\triangle_{4}}$ & 12 & 1980 & 3.30 & 15 & 2211 & 3.68 \\
$\sigma_{\triangle_{4}}^{2}$ & 6 & 1701 & 2.84 & 8 & 1656 & 2.76 \\
$\mu_{k}$ & 6 & 1432 & 2.39 & 8 & 1456 & 2.43 \\
$\sigma_{k}^{2}$ & 4 & 1236 & 2.06 & 4 & 771 & 1.28 \\
$\mu_{\delta}$ & 18 & 3090 & 5.15 & 24 & 4912 & 8.19 \\
$\sigma_{\delta}^{2}$ & 15 & 2499 & 4.16 & 30 & 6955 & 11.60 \\
\hline
\end{tabular}

Table 2: Diagnostic statistics for global parameters in BHM for $e_{0}^{N S}$. The quantities shown are defined as in Table 1 .

\begin{tabular}{|c|c|c|c|c|c|c|}
\hline Parameters & Burn1 & Size1 & DF1 & Burn2 & Size2 & DF2 \\
\hline$\mu_{a_{1}}$ & 164 & 14734 & 24.60 & 3 & 703 & 1.17 \\
$\sigma_{a_{1}}^{2}$ & 2 & 596 & 0.99 & 3 & 648 & 1.08 \\
$\mu_{a_{2}}$ & 2 & 572 & 0.95 & 81 & 10795 & 18.00 \\
$\sigma_{a_{2}}^{2}$ & 3 & 648 & 1.08 & 3 & 648 & 1.08 \\
$\mu_{a_{3}}$ & 2 & 572 & 0.95 & 7 & 1179 & 1.96 \\
$\sigma_{a_{3}}^{2}$ & 2 & 596 & 0.99 & 2 & 621 & 1.03 \\
$\mu_{a_{4}}$ & 3 & 703 & 1.17 & 6 & 1005 & 1.68 \\
$\sigma_{a_{4}}^{2}$ & 3 & 675 & 1.12 & 5 & 867 & 1.44 \\
$\mu_{w}$ & 3 & 648 & 1.08 & 2 & 596 & 0.99 \\
$\sigma_{w}^{2}$ & 2 & 572 & 0.95 & 2 & 596 & 0.99 \\
$\mu_{z}$ & 3 & 662 & 1.10 & 2 & 572 & 0.95 \\
$\sigma_{z}^{2}$ & 2 & 596 & 0.99 & 3 & 689 & 1.15 \\
\hline
\end{tabular}



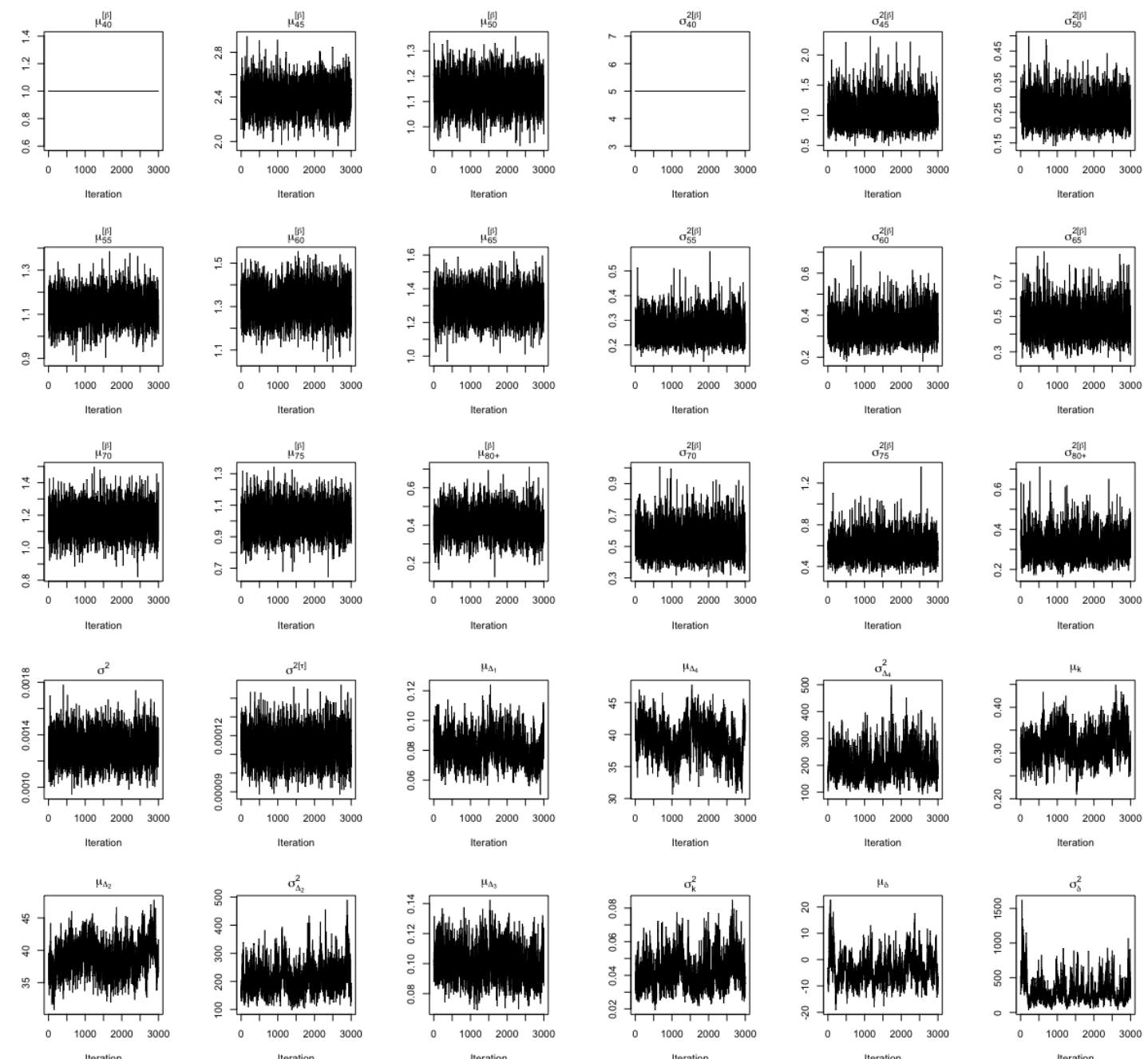

Figure 1: Traceplots for the hyperparameters in BHM for ASSAF. 

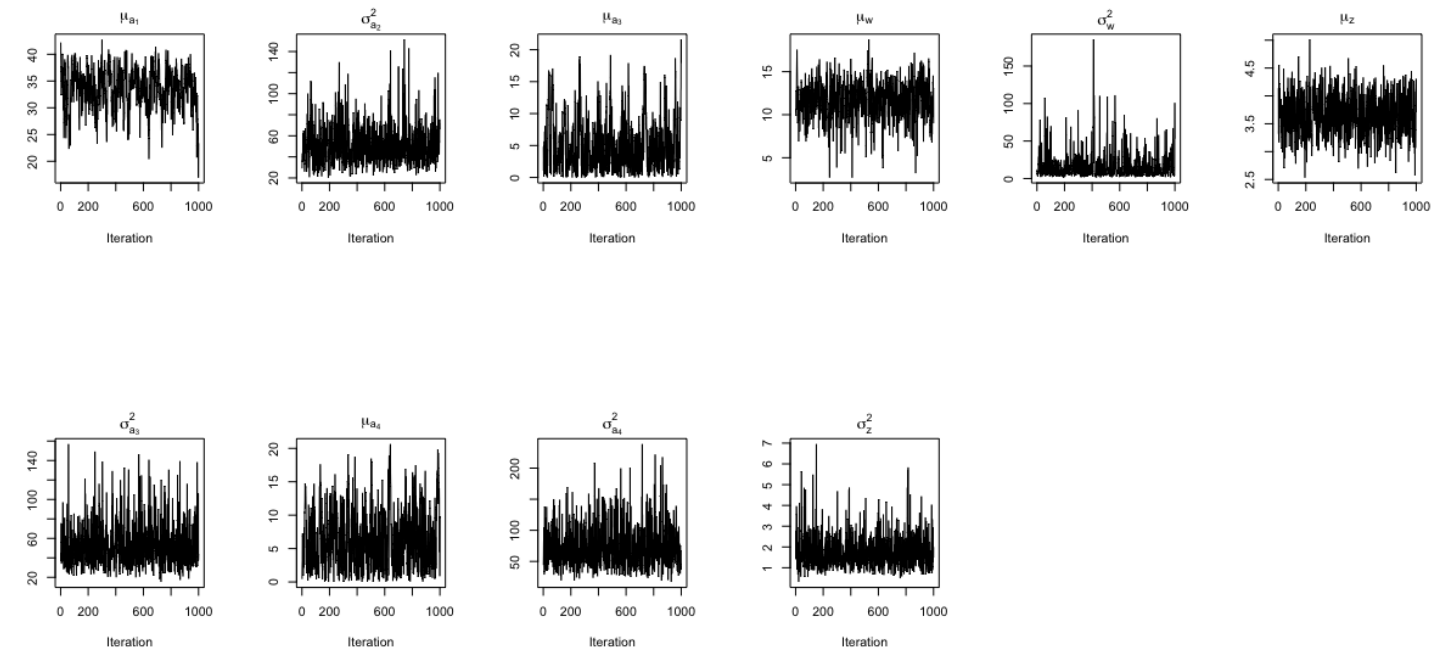

Figure 2: Traceplots for the hyperparameters in BHM for $e_{0}^{N S}$. 


\section{Life Expectancy Forecast Plots for Over 60 Countries}
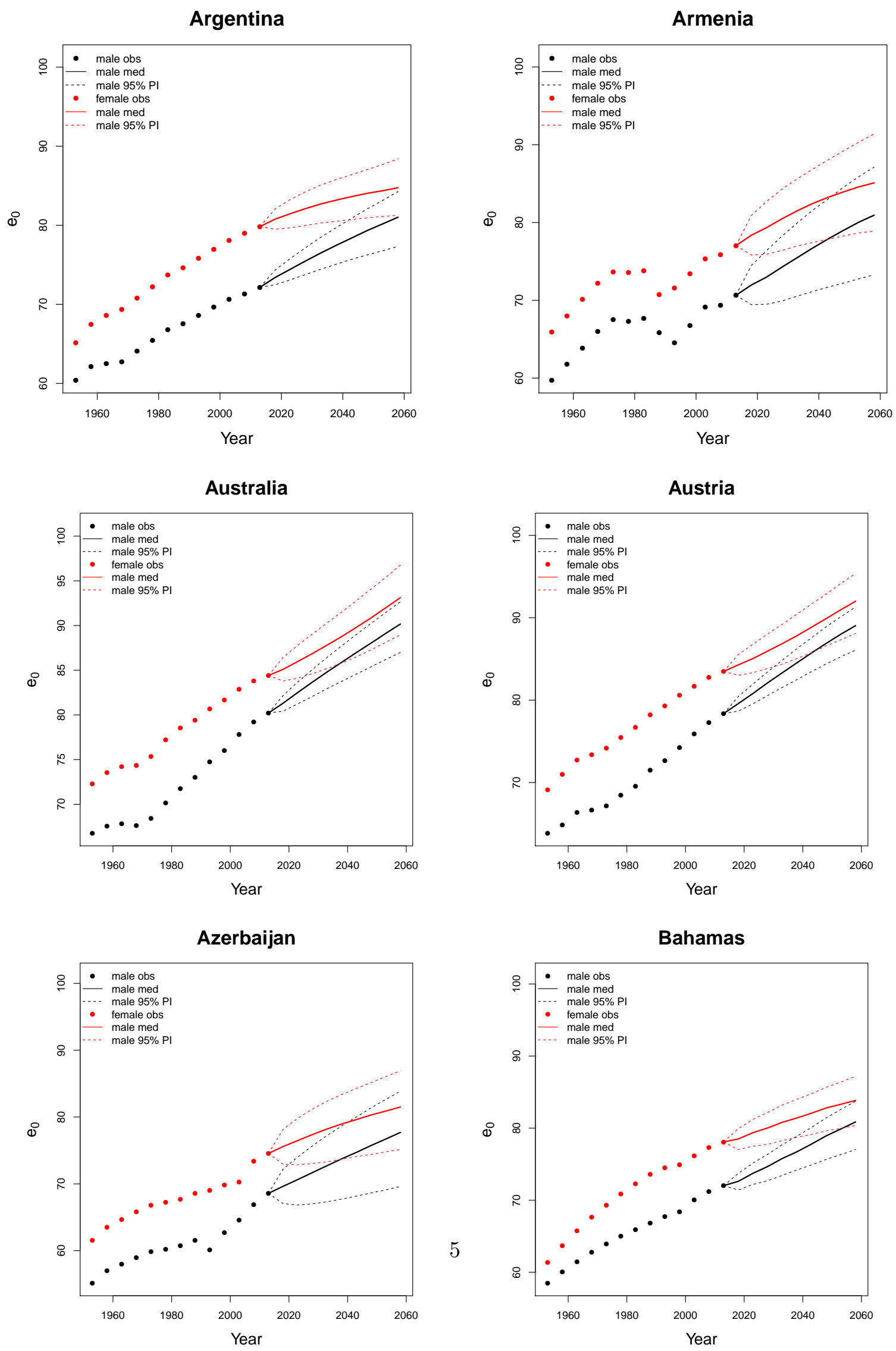
Bahrain

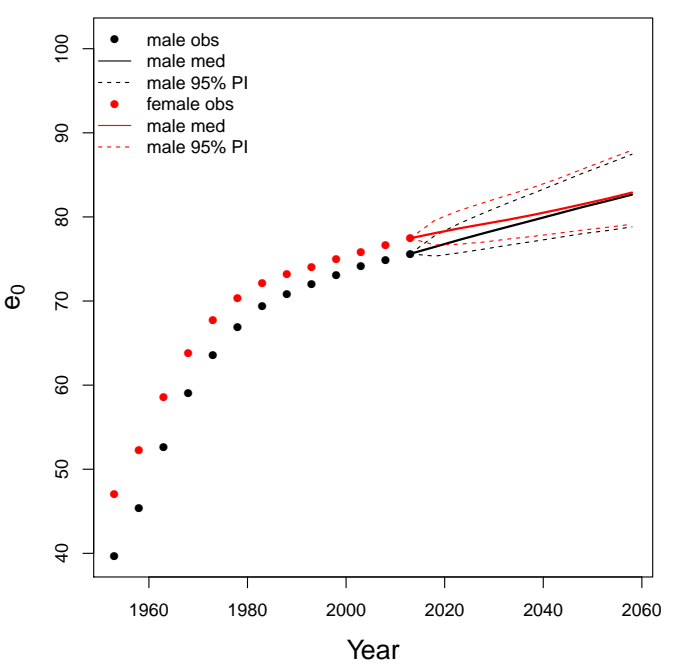

\section{Belgium}

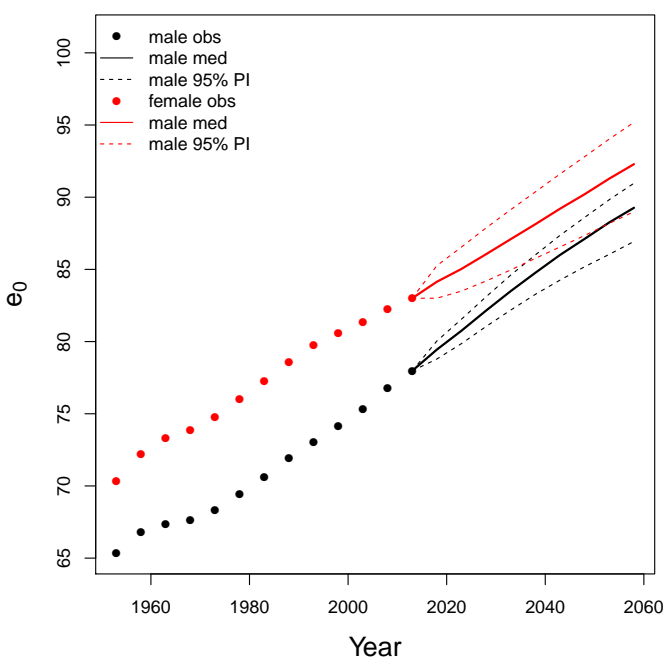

Canada

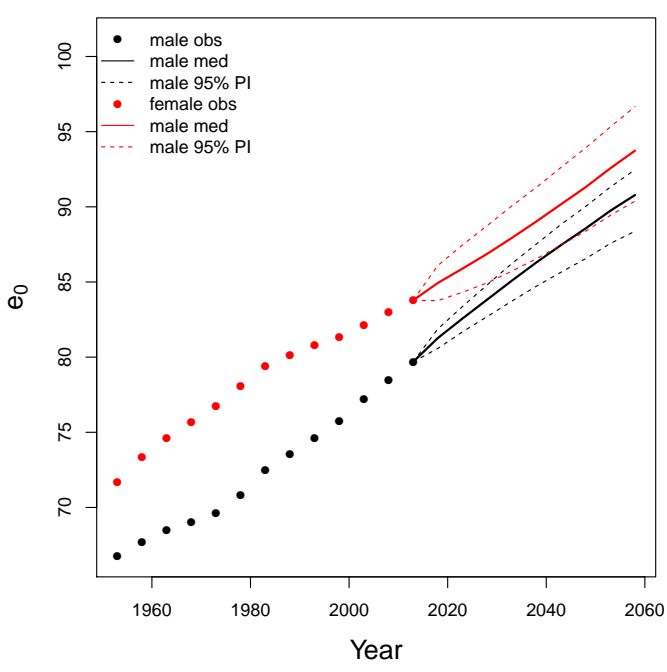

Belarus

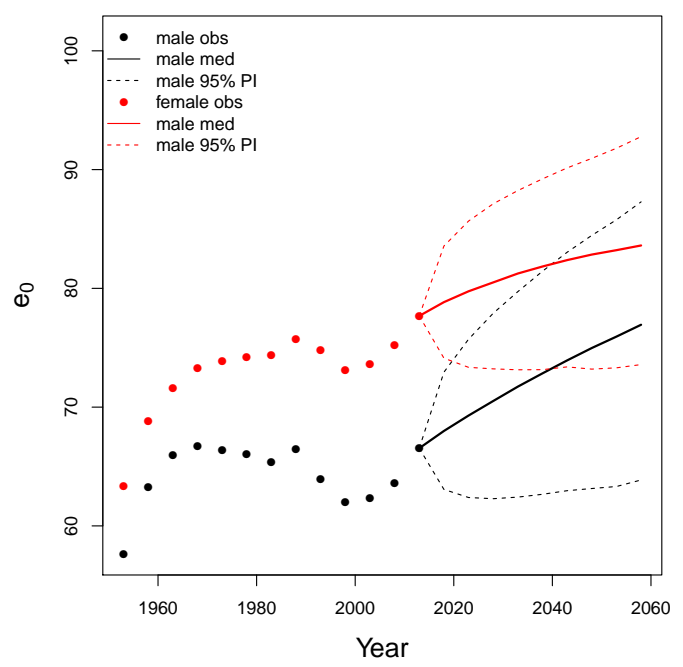

\section{Brazil}

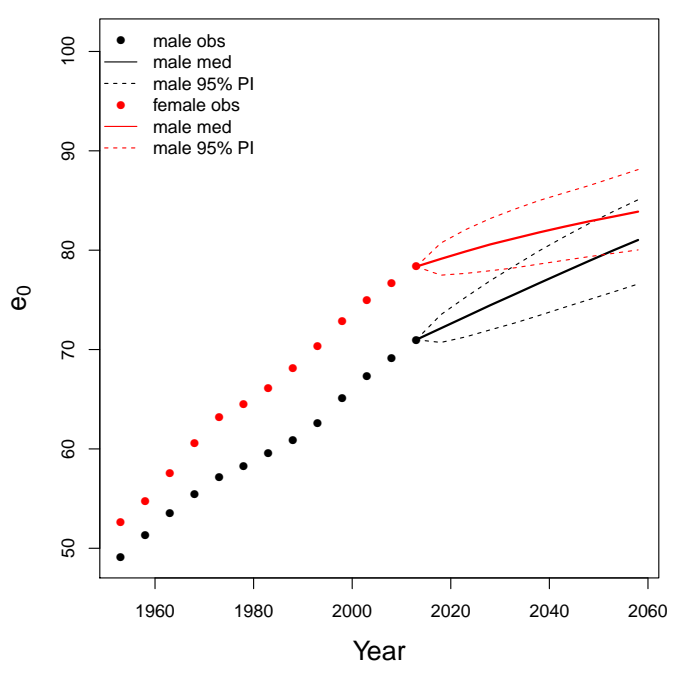

Chile

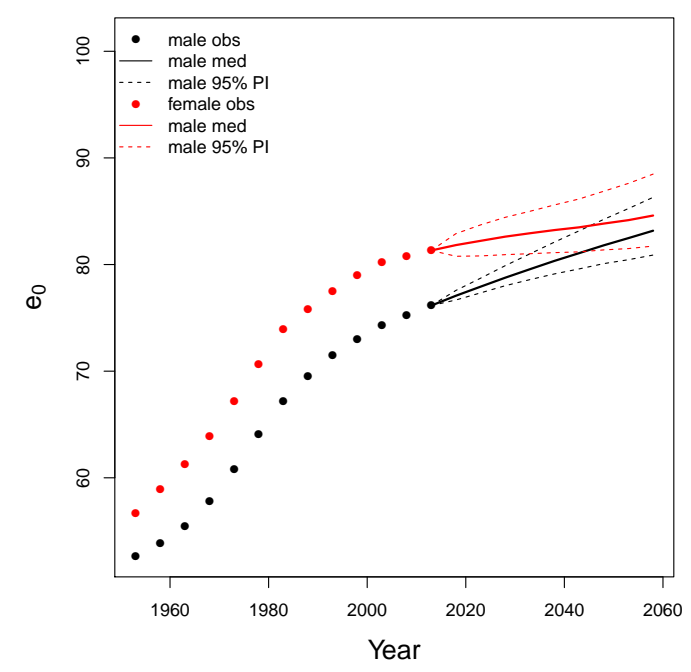



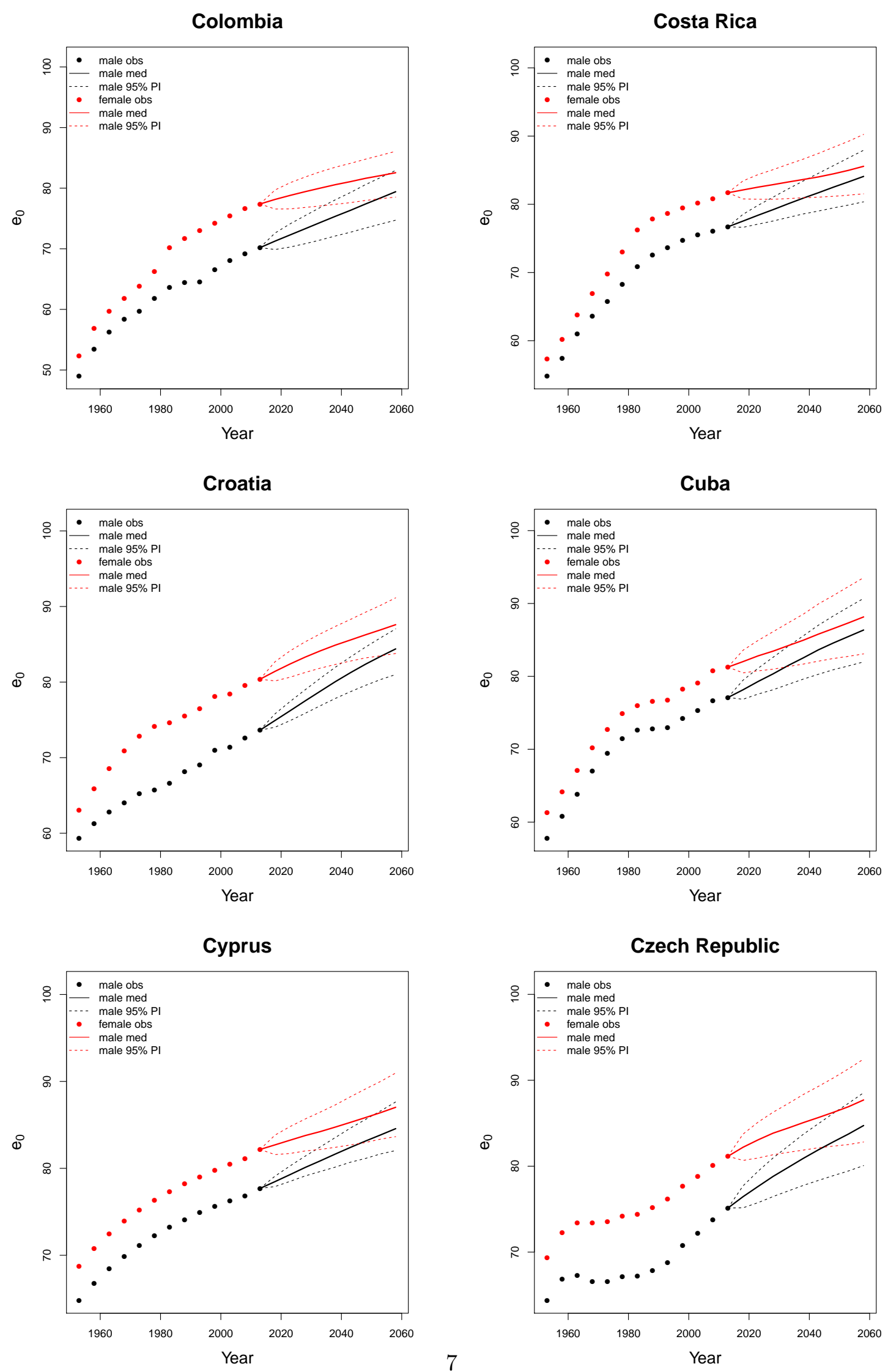


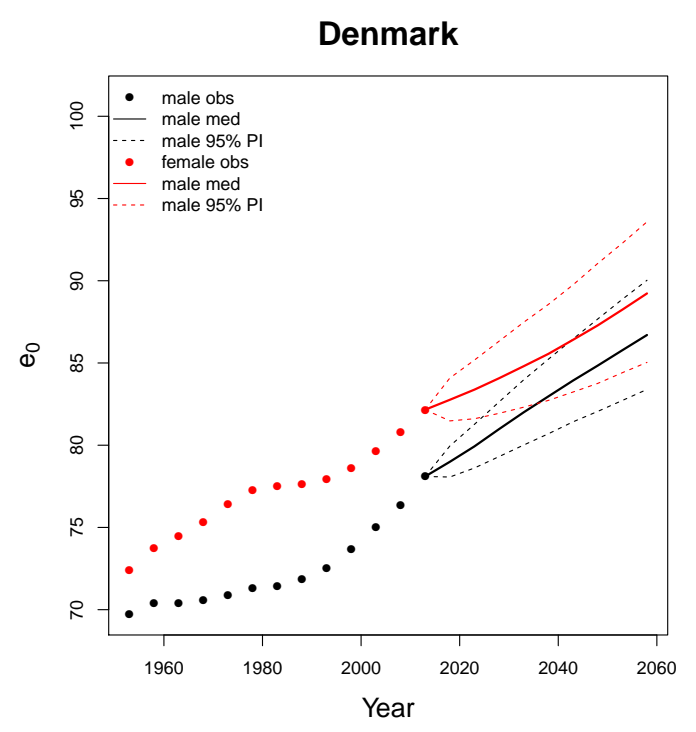

Finland

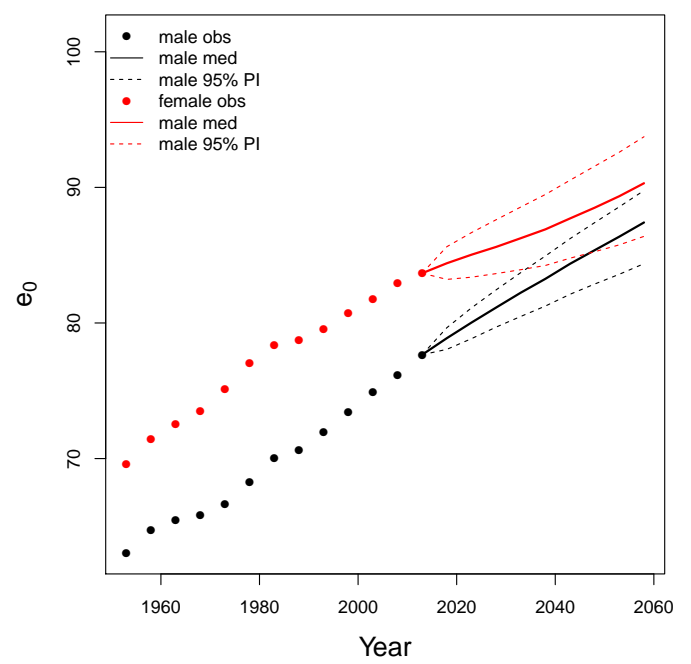

\section{Germany}

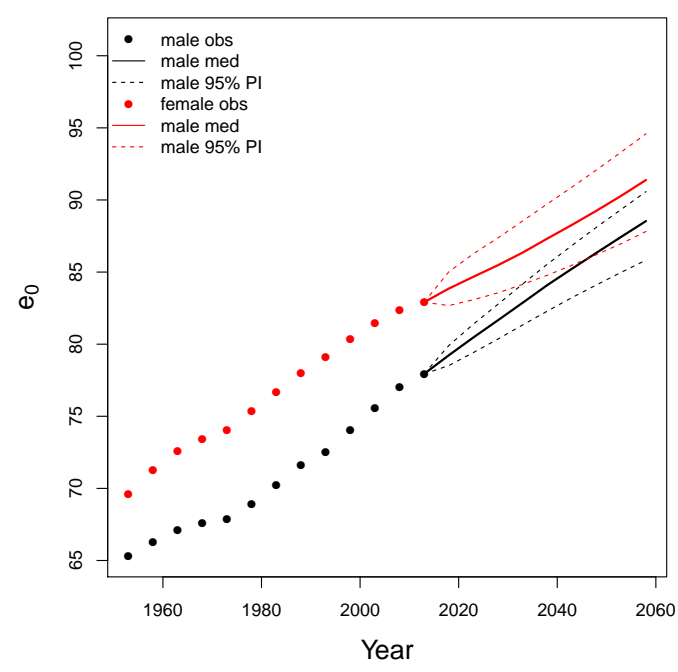

Estonia

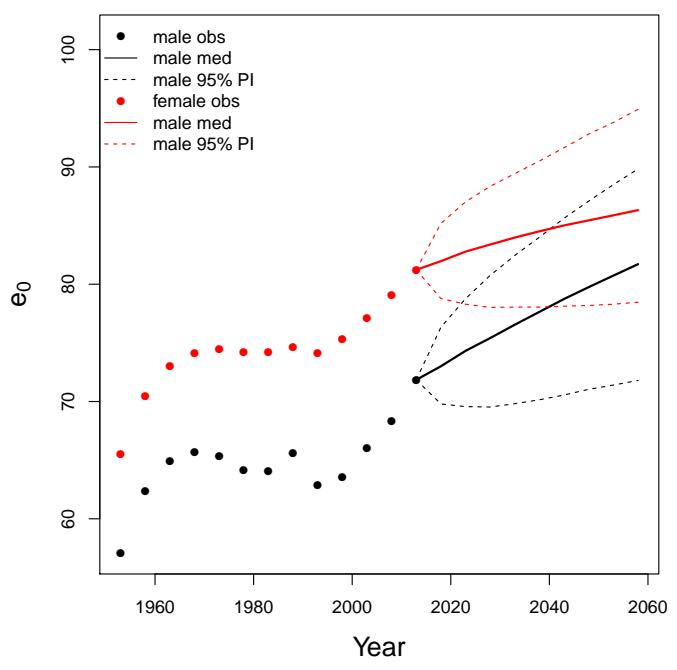

France

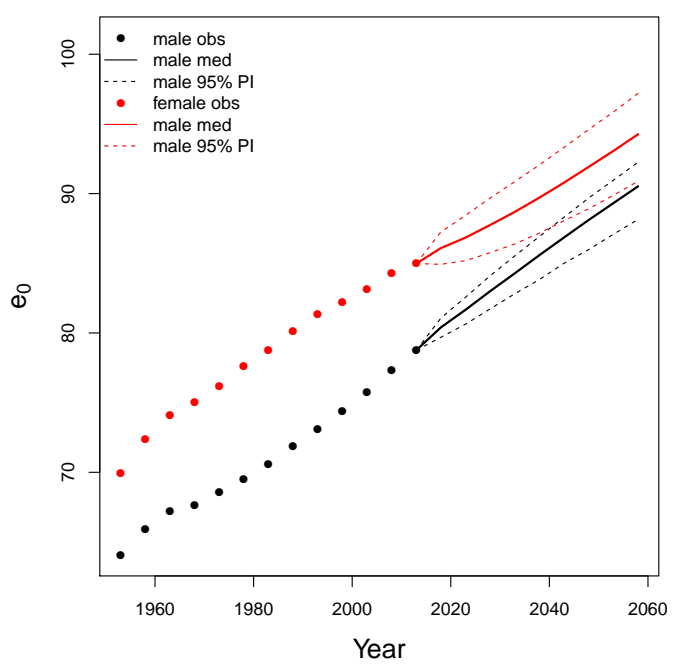

Greece

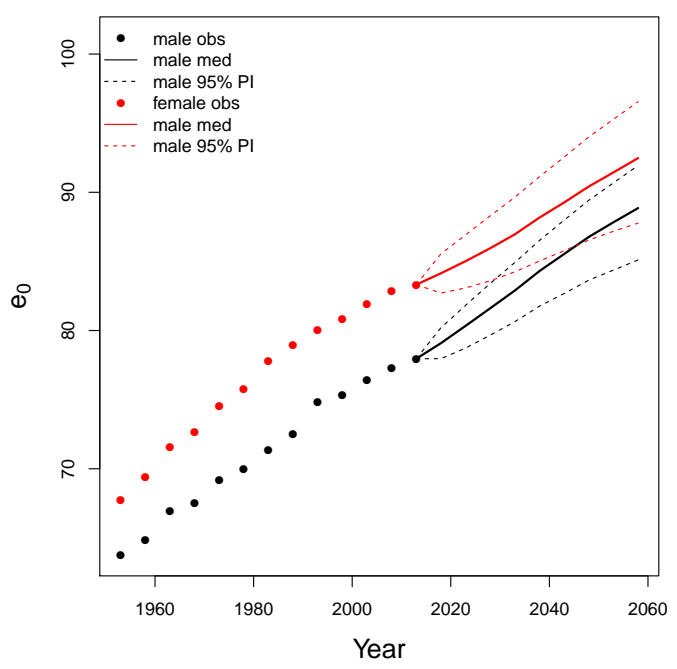




\section{Hong Kong SAR}

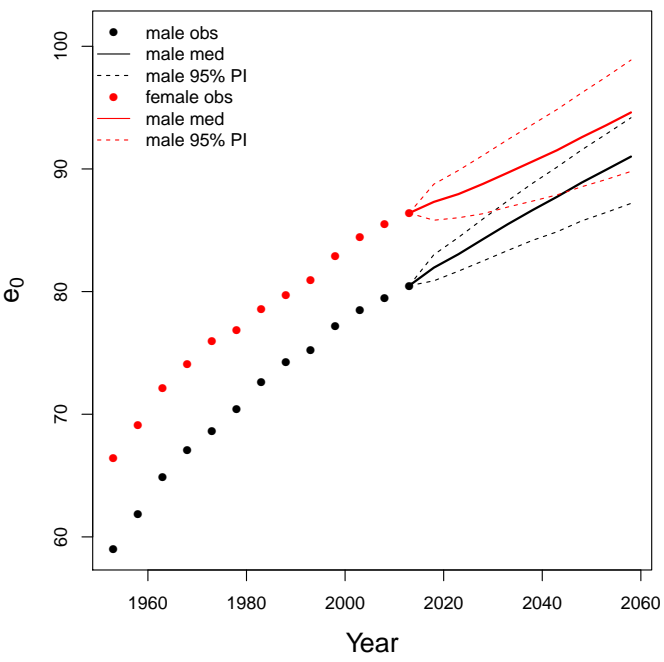

Iceland

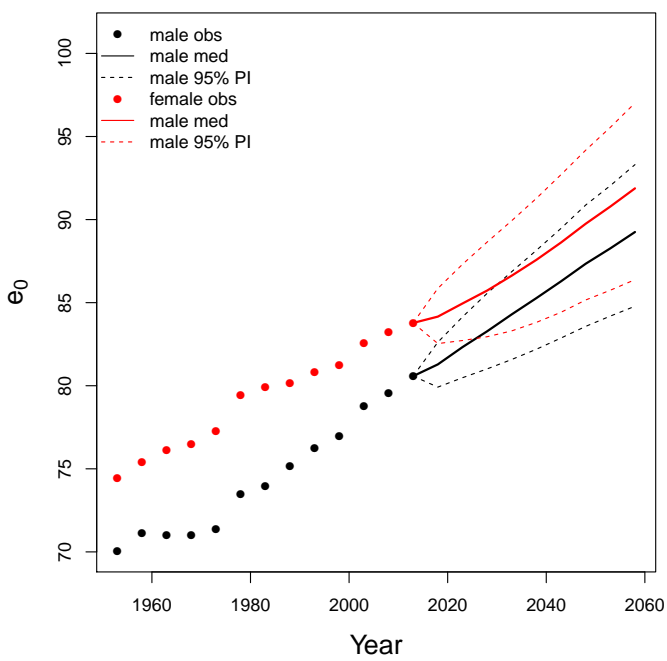

Israel

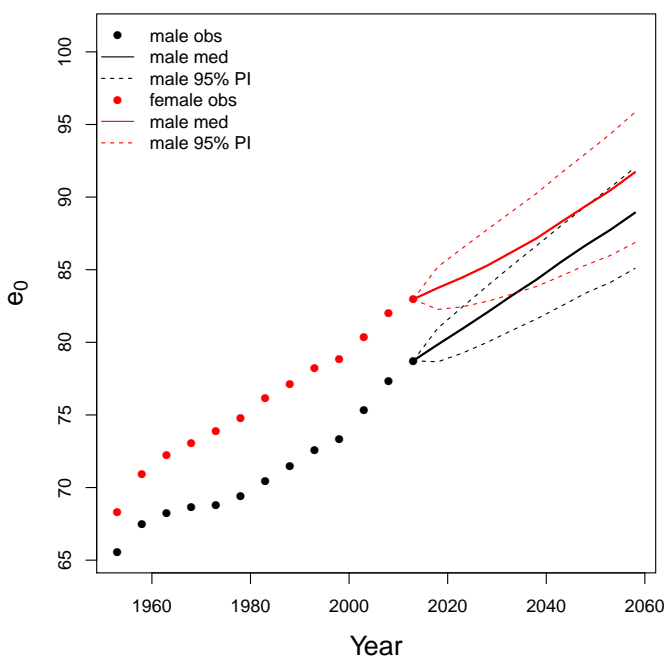

Hungary

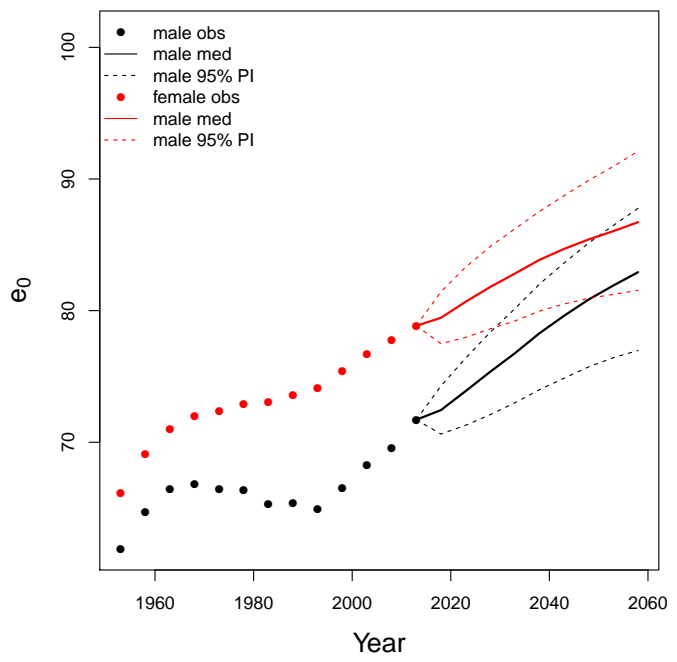

Ireland

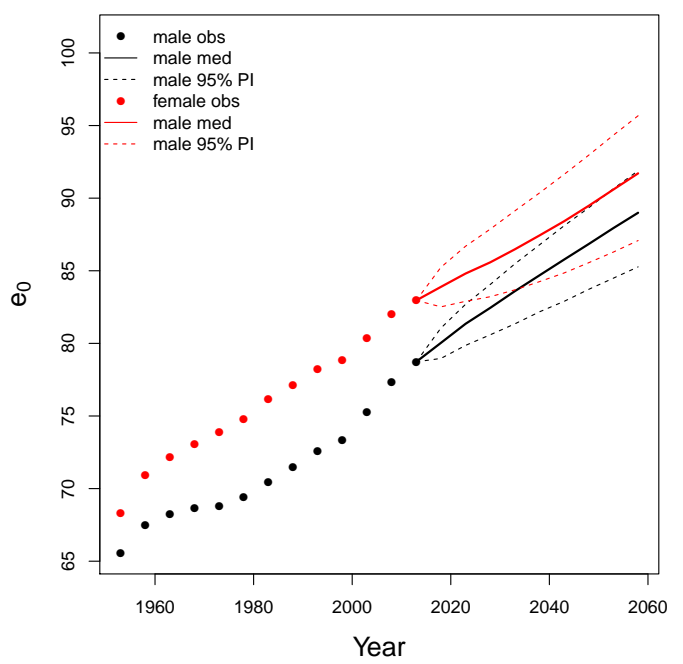

Italy

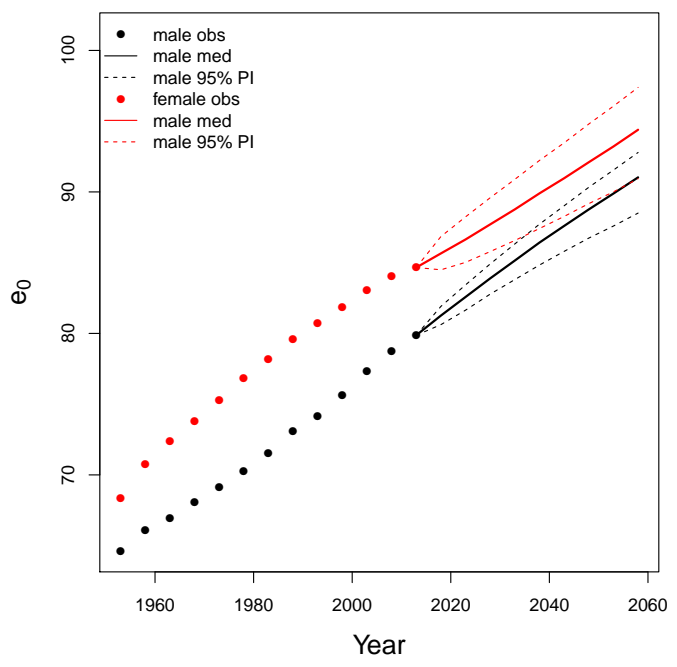



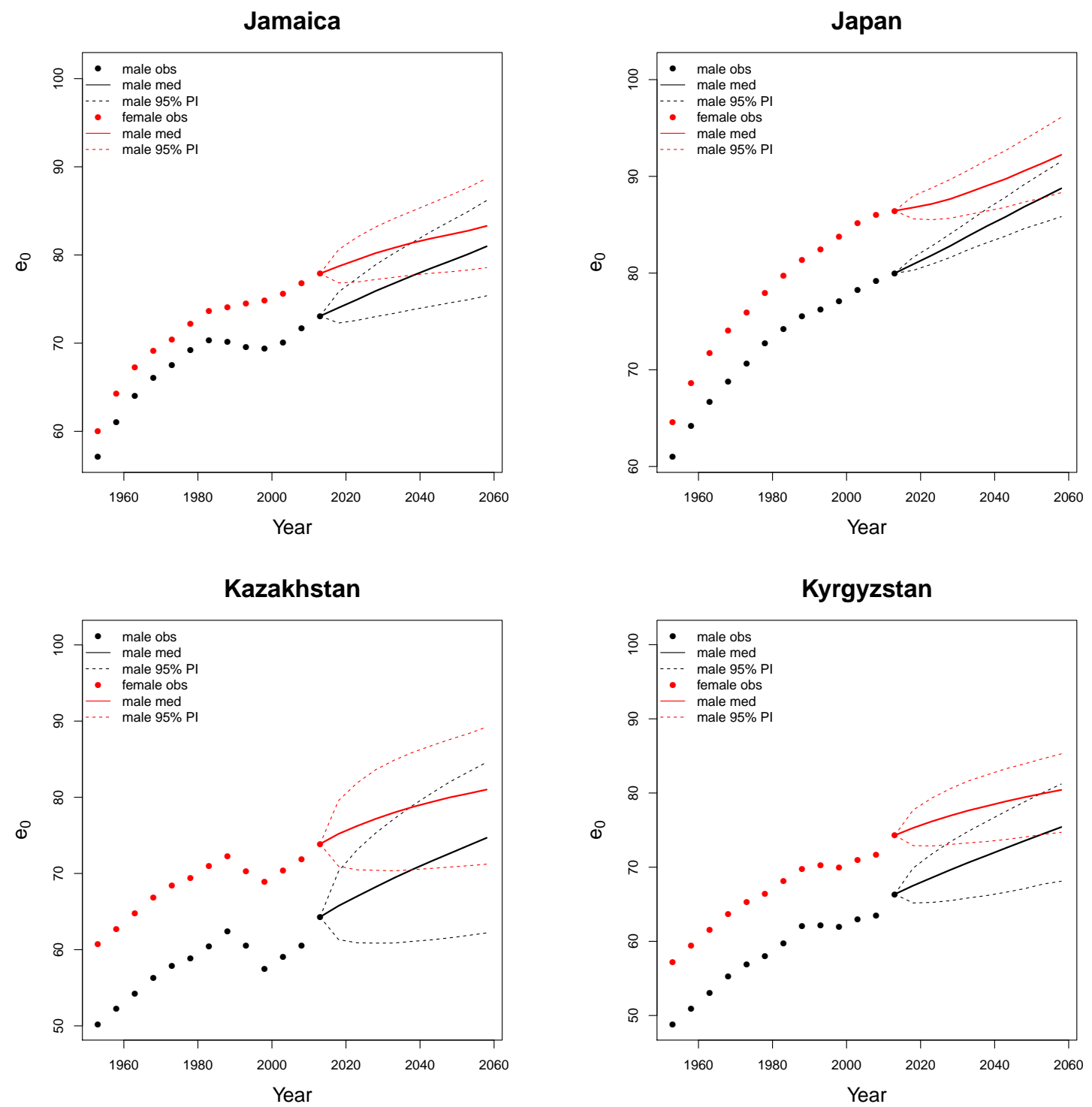

\section{Latvia}
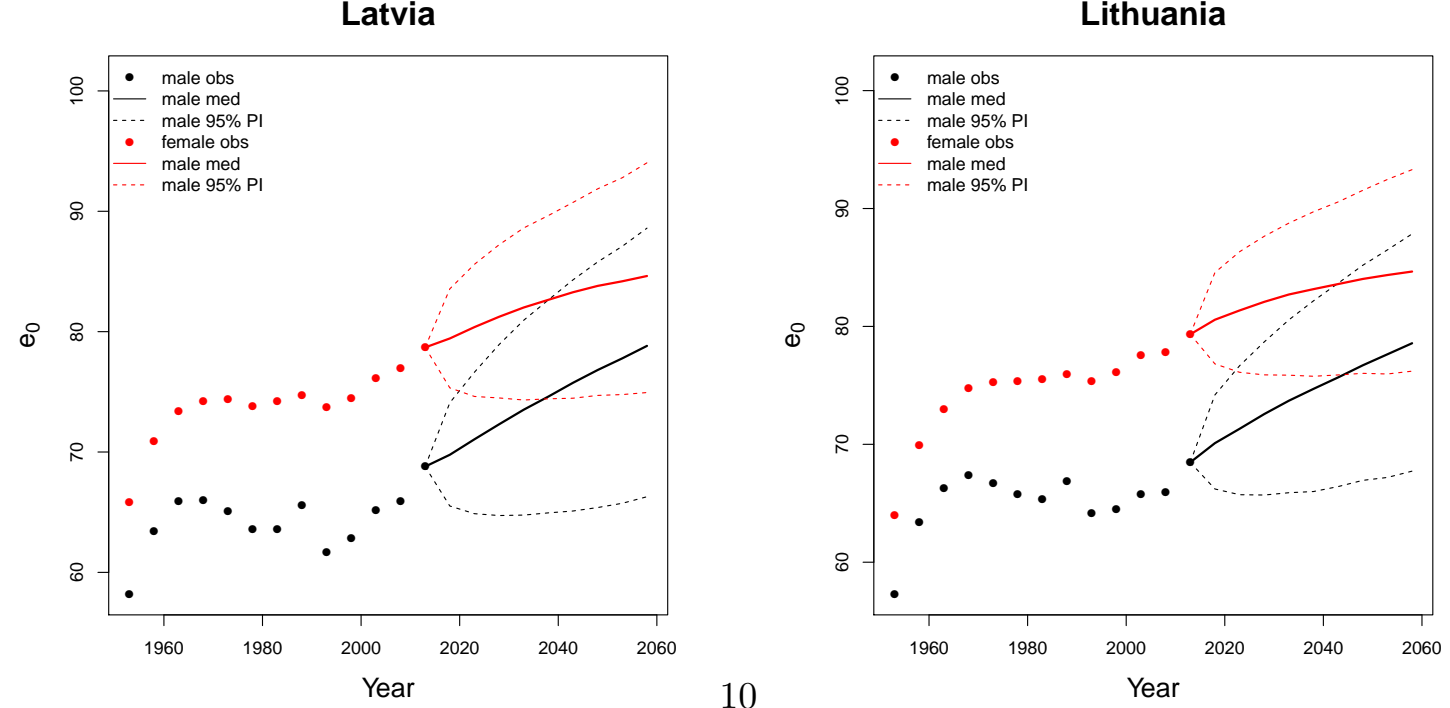

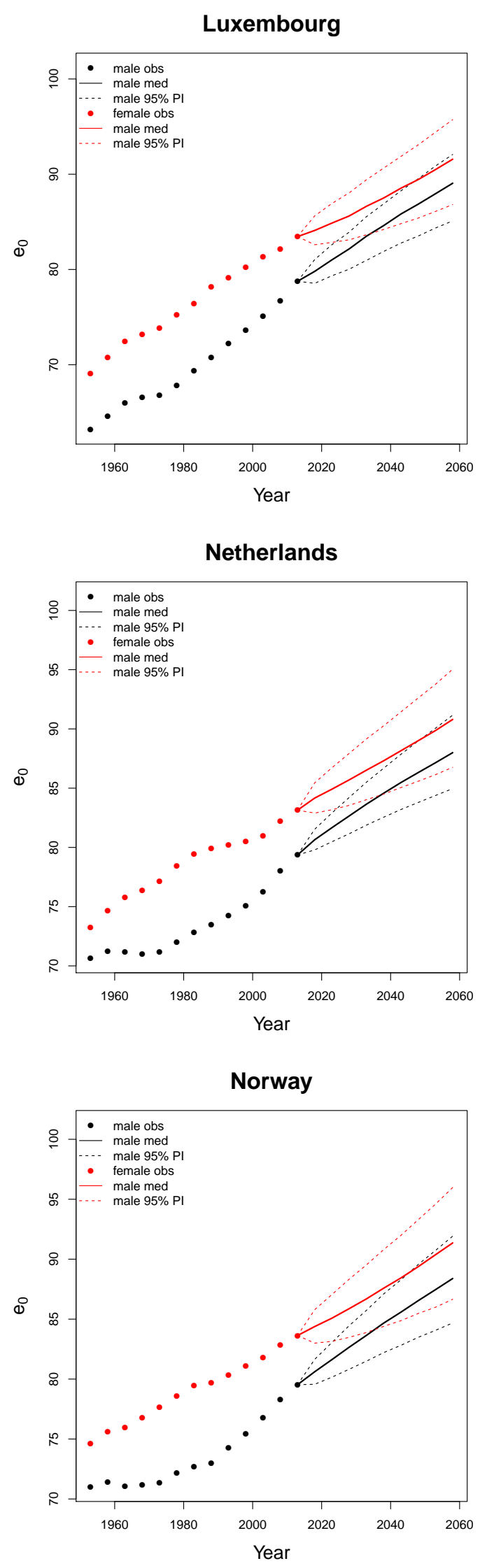
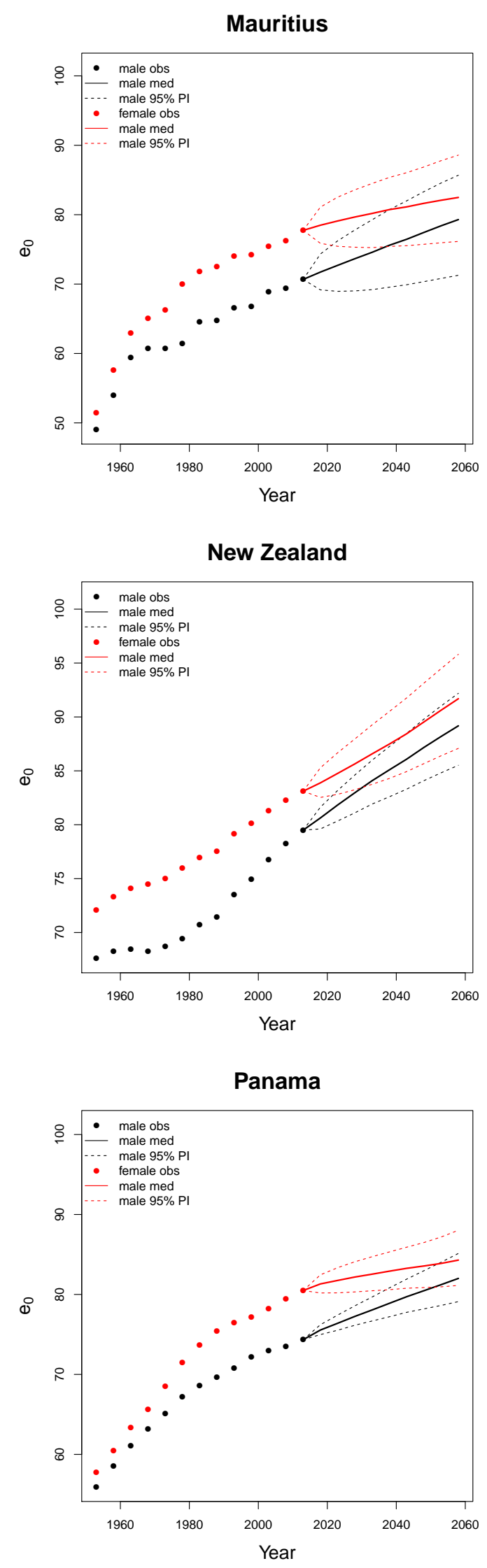

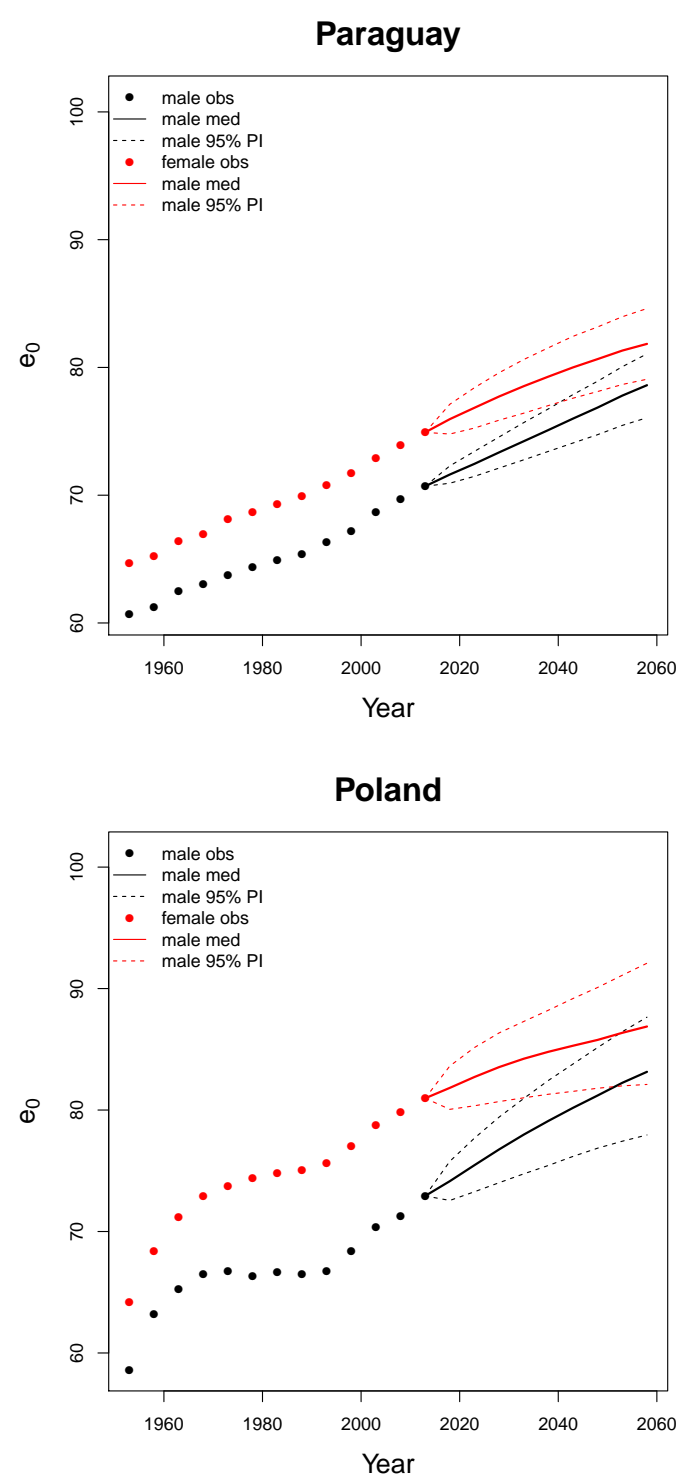

Puerto Rico

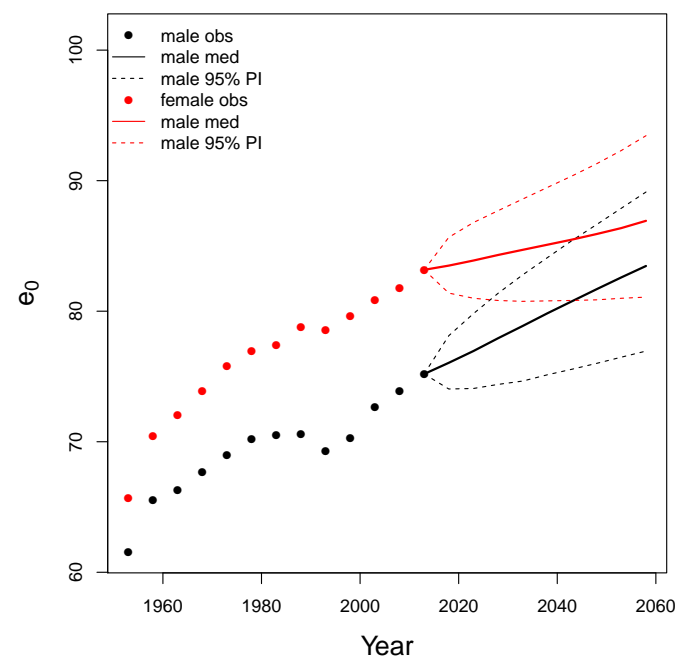

Philippines

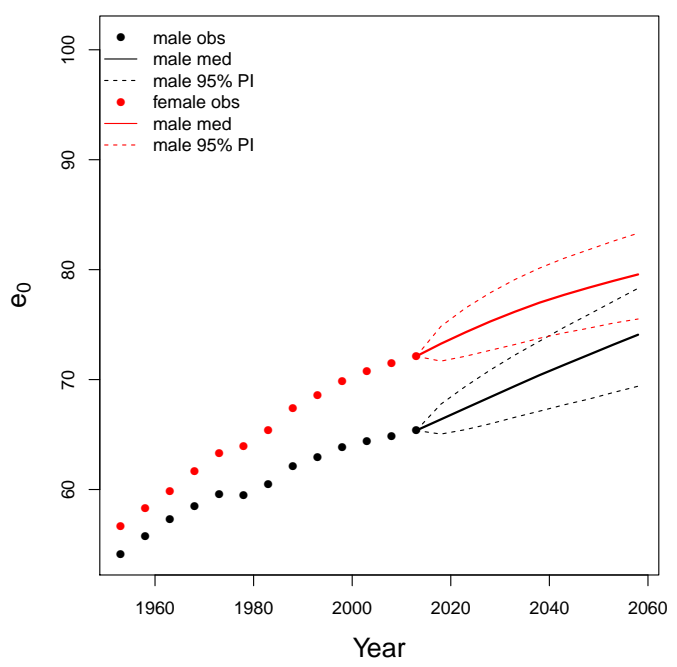

Portugal

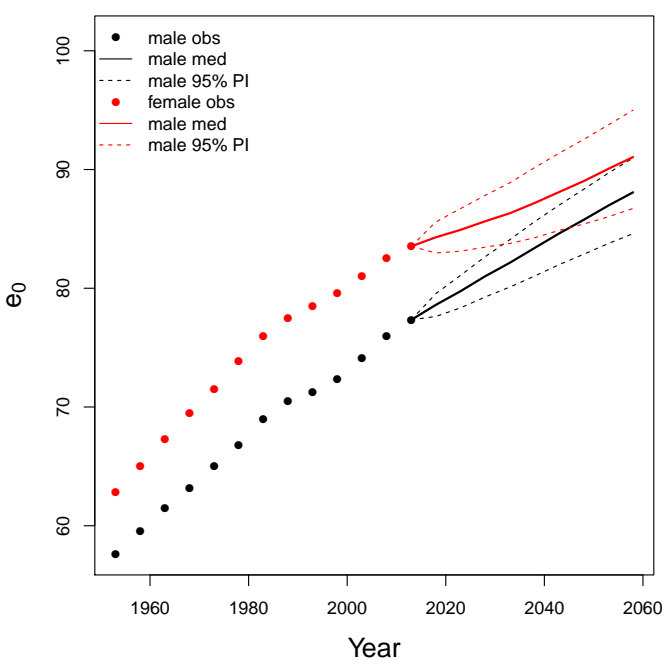

Republic of Korea

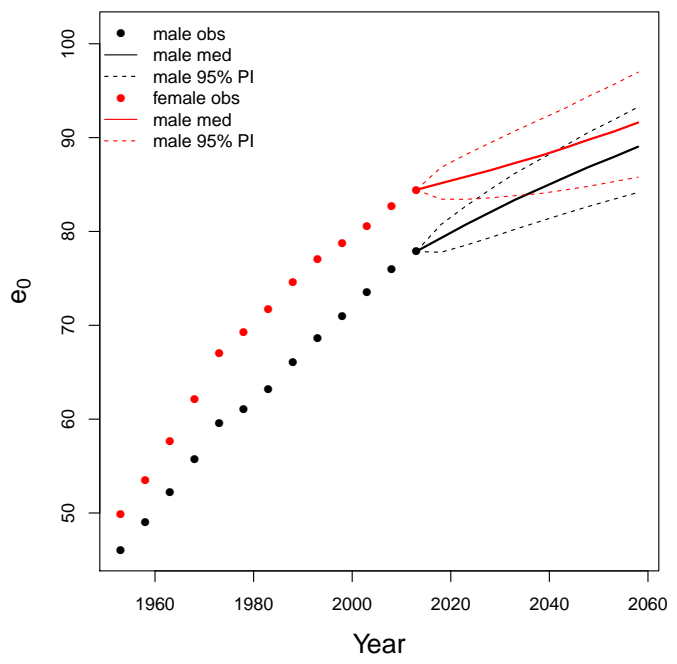




\section{Romania}

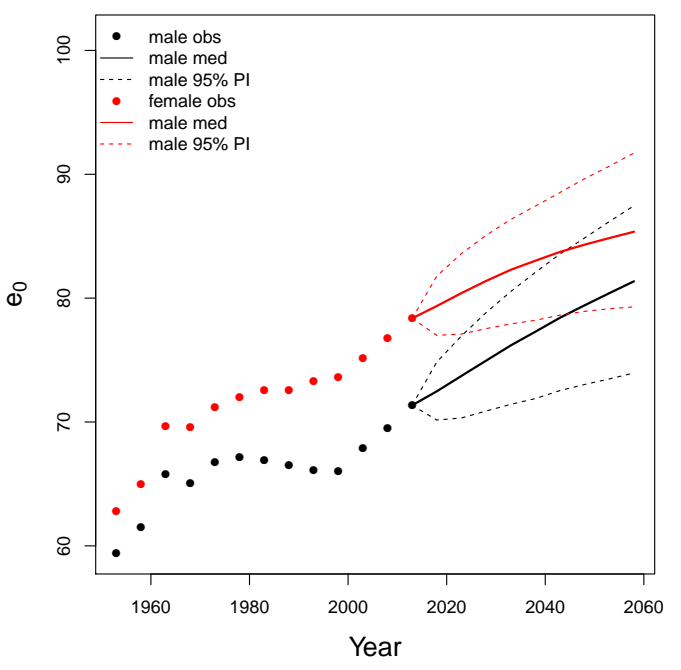

\section{Serbia}

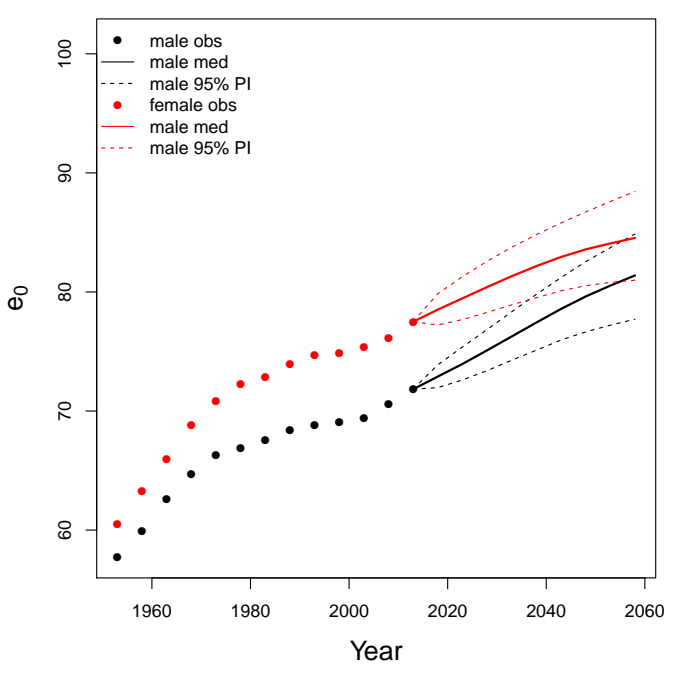

Slovakia

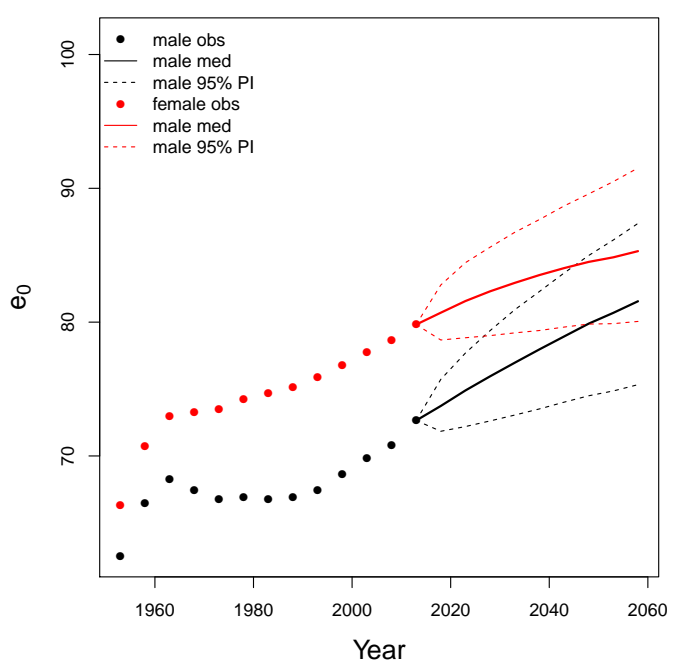

\section{Russian Federation}

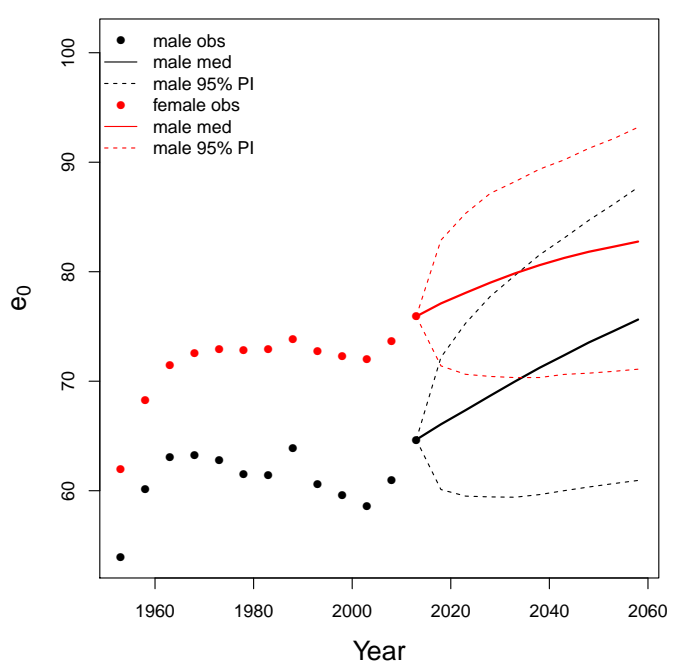

Singapore

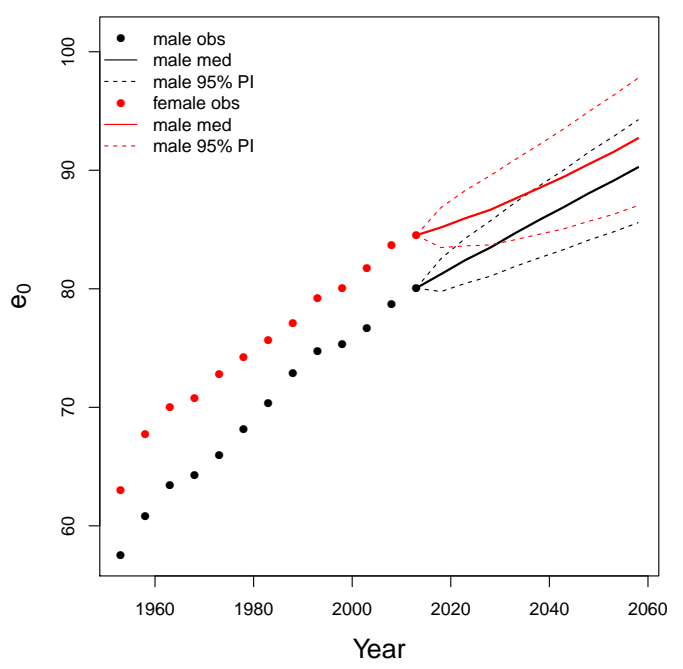

Slovenia

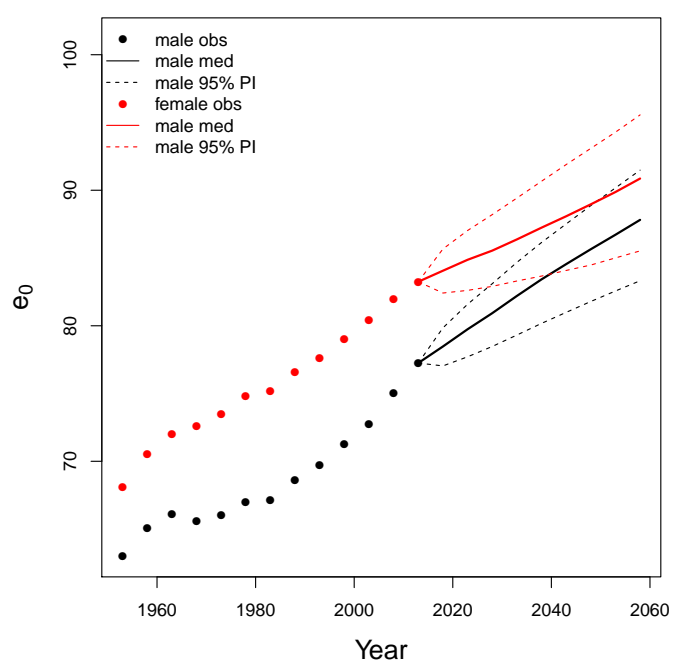



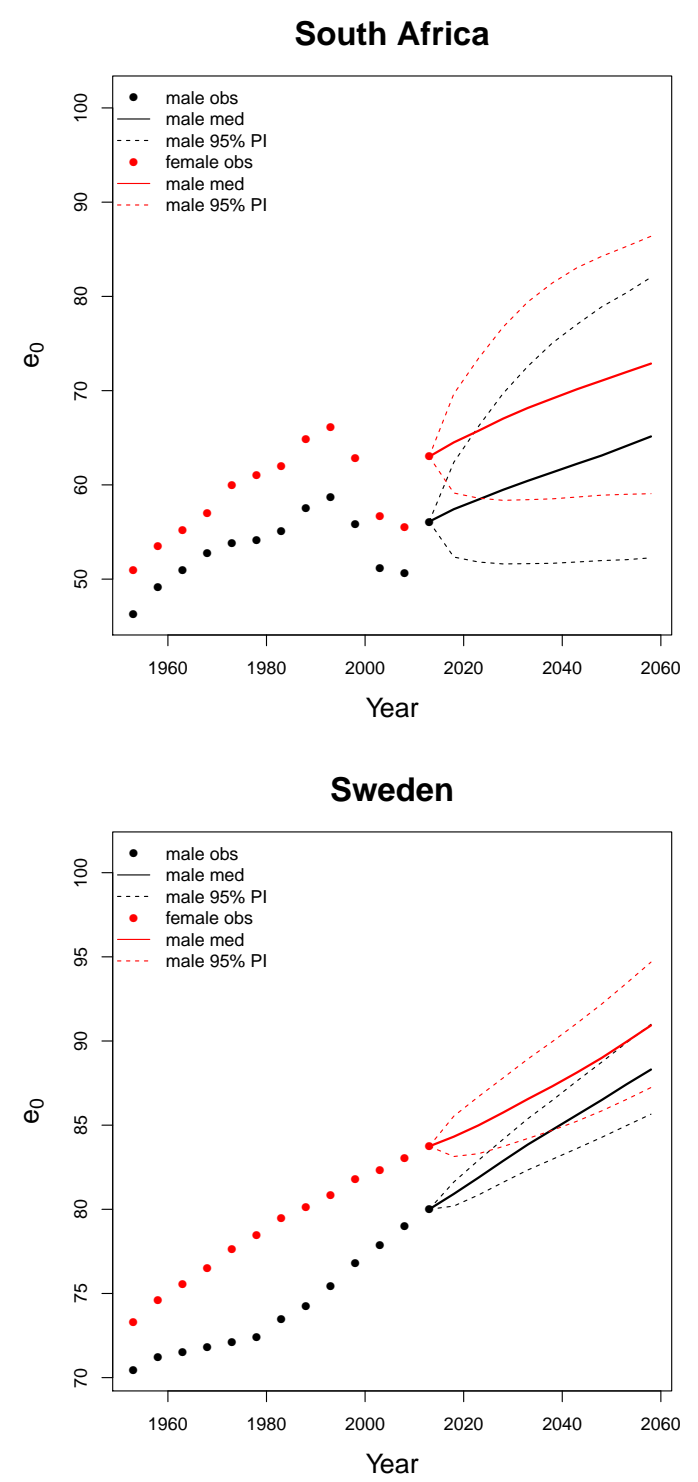

Tajikistan

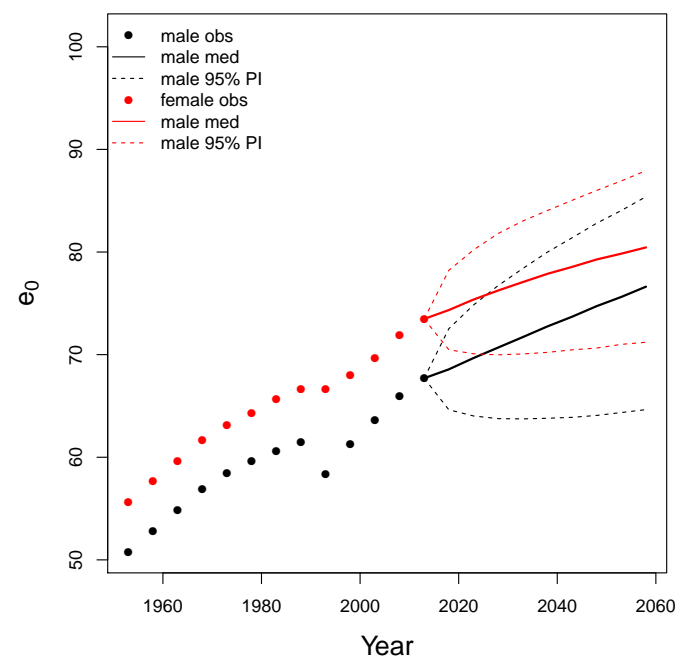

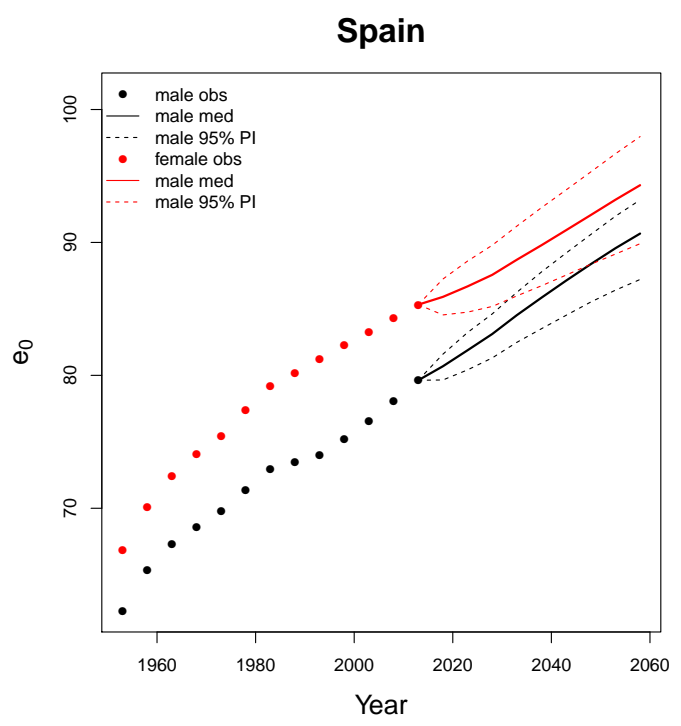

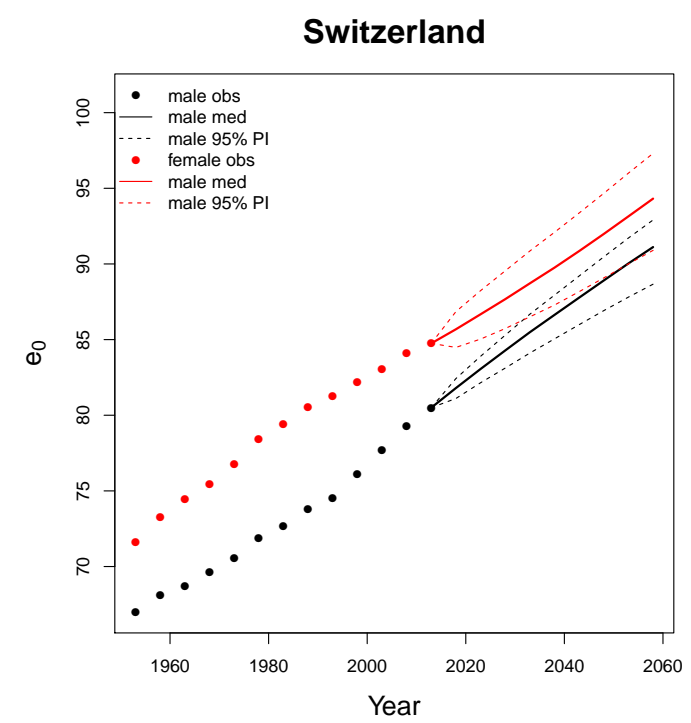

TFYR Macedonia

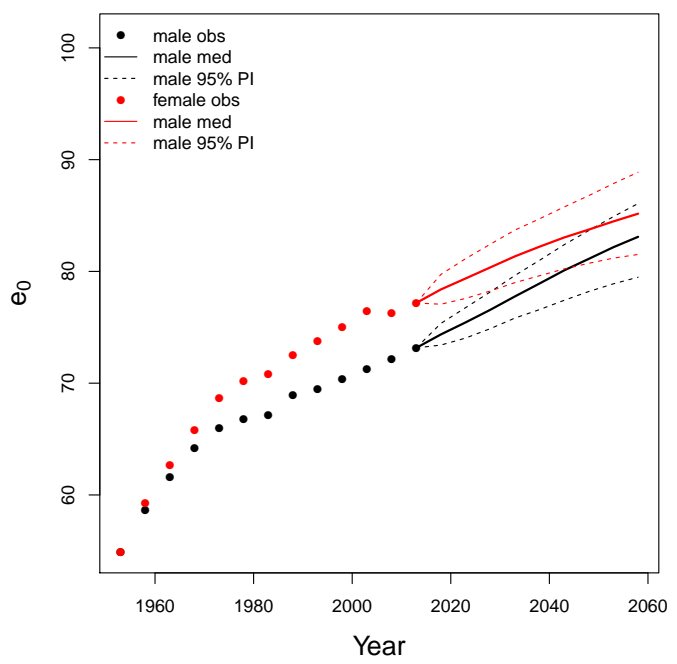




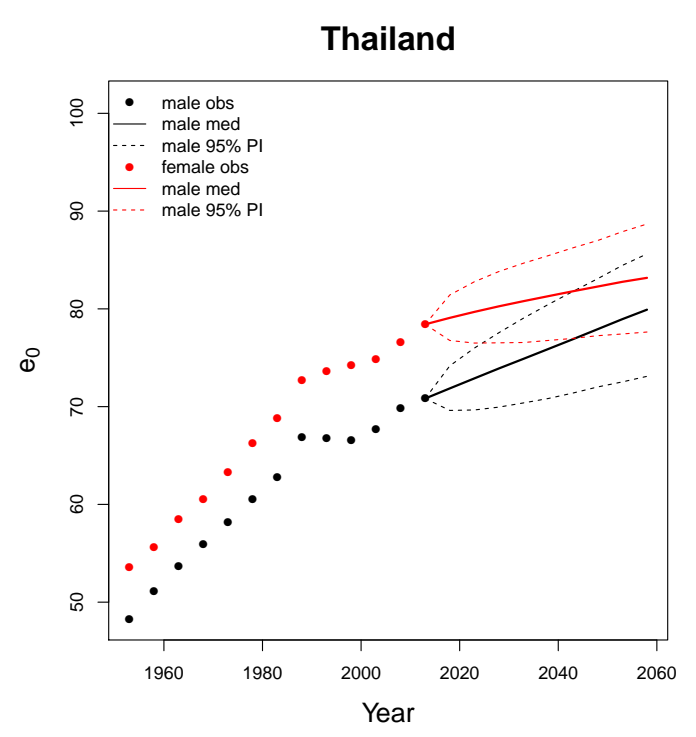

\section{Turkmenistan}

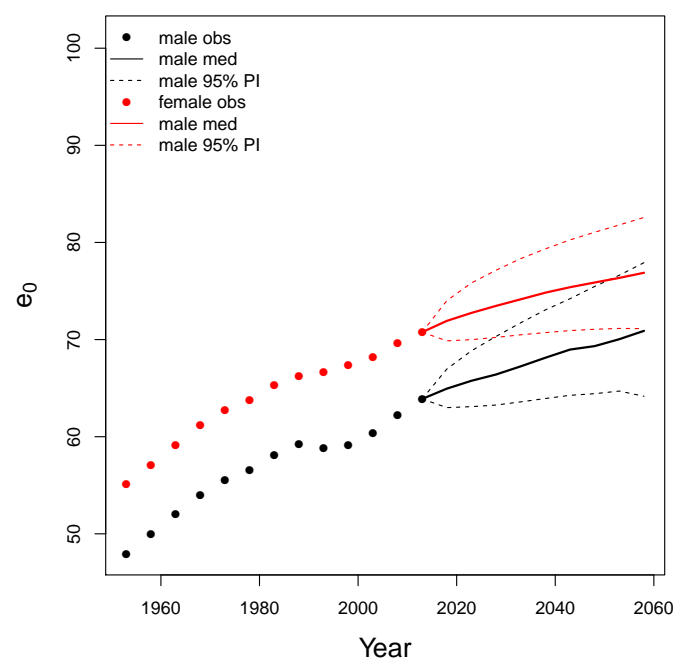

United Kingdom

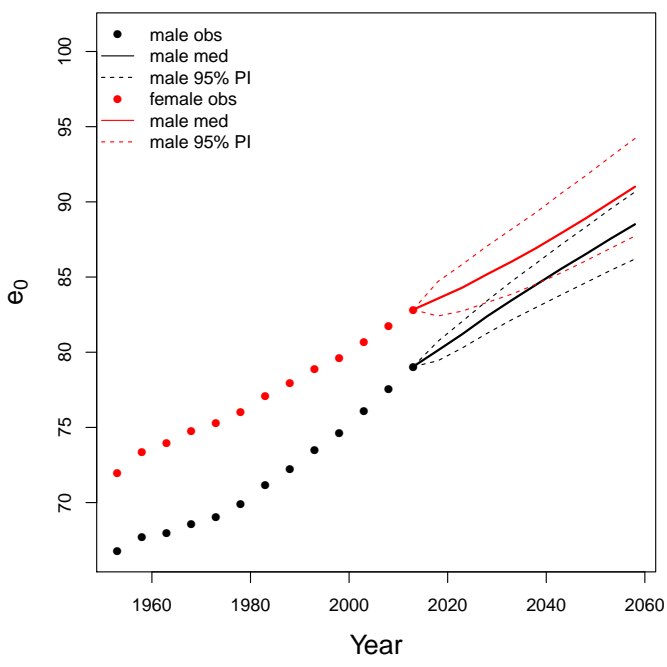

Trinidad and Tobago

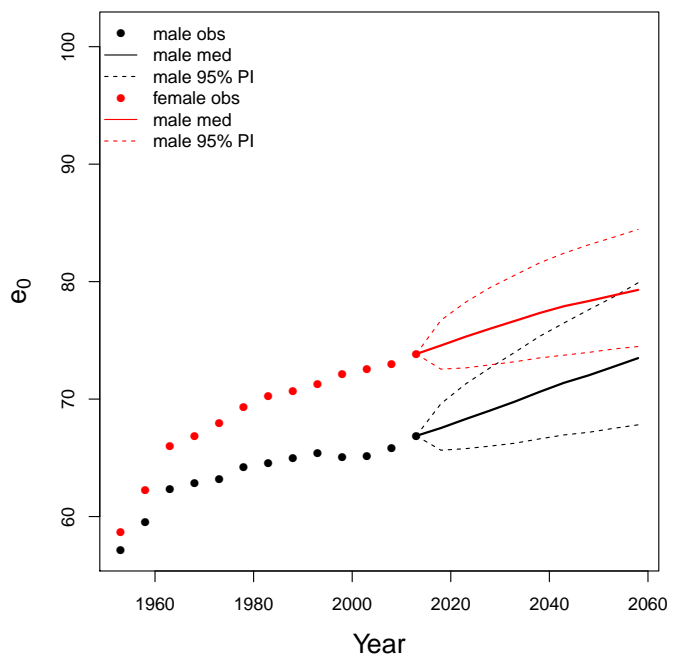

Ukraine

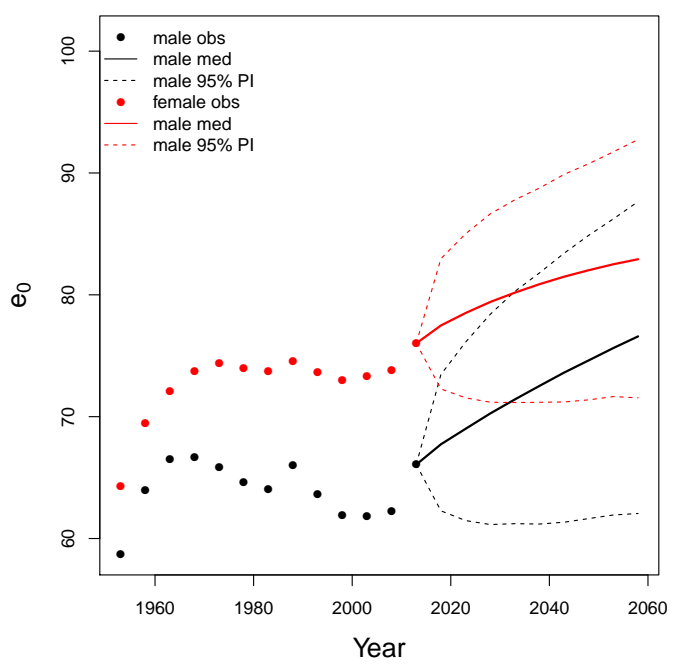

United States of America

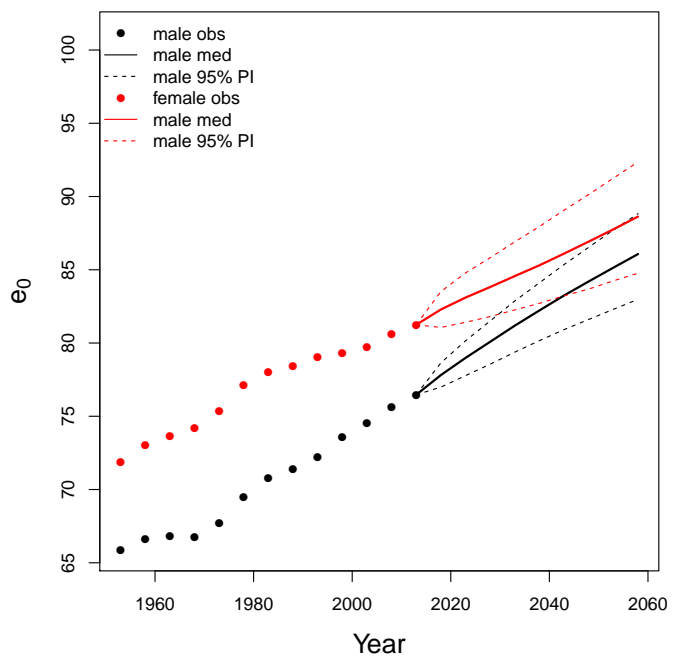



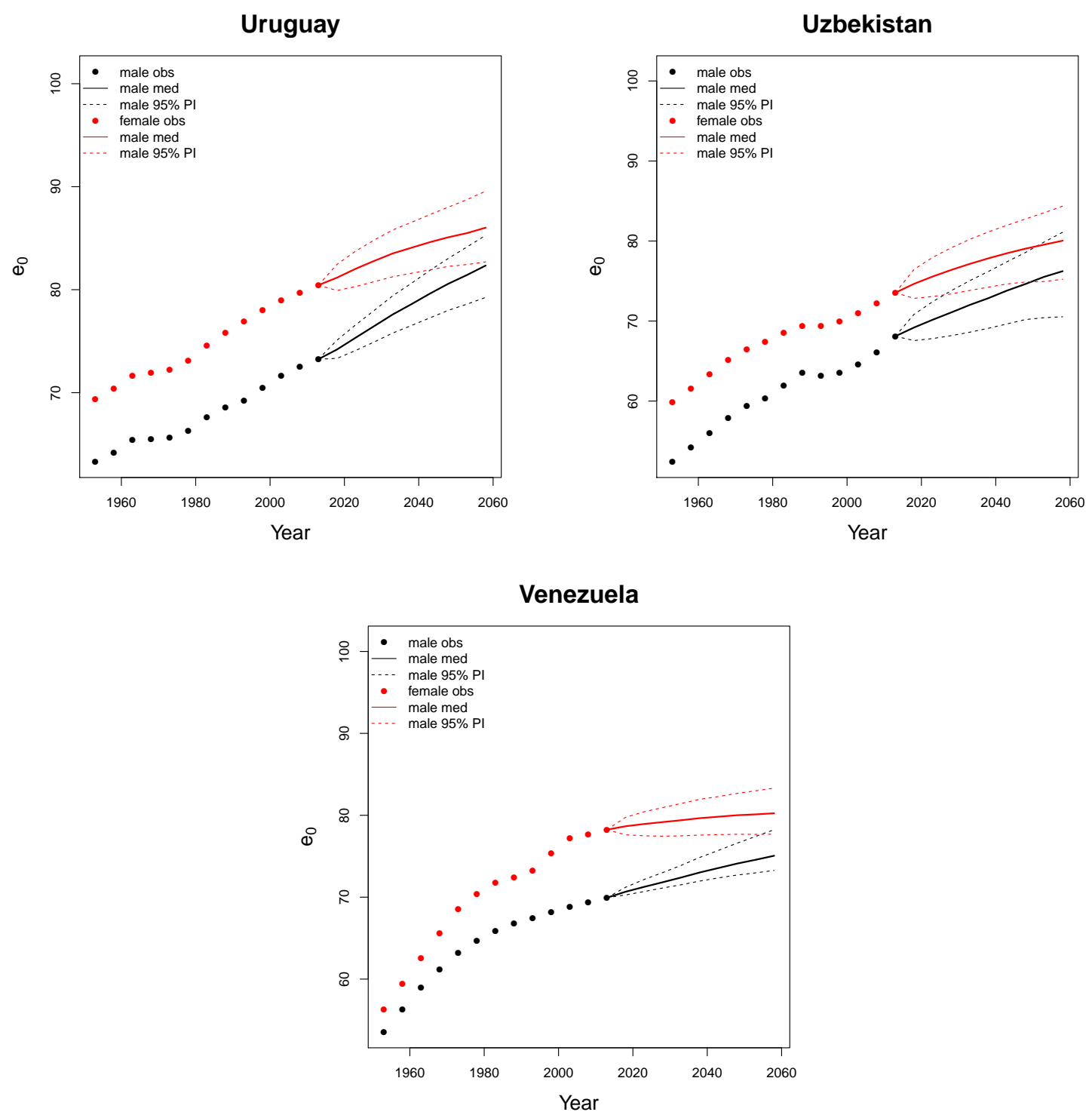

\section{References}

Raftery, A. E. and Lewis, S. M. (1992). One long run with diagnostics: Implementation strategies for Markov chain Monte Carlo. Statistical Science, 7(4):493-497. 